\title{
Modelling of a Solid Fuel Combustion Chamber of a Ramjet Using a Multi-block Domain Decomposition Technique *
}

\author{
P. J. Coelho ${ }^{(1)}$, N. Duic ${ }^{(2)}$, C. Lemos ${ }^{(1)}$, M. G. Carvalho ${ }^{(1)}$ \\ (1) Instituto Superior Técnico, Departamento de Engenharia Mecânica, \\ Pavilhão de Maquinas 1" andar., Av. Rovisco Pais, 1096 Lisboa Codex, Portugal. \\ (2) Faculty of Mechanical Engineering and Naval Architecture, \\ University of Zagreb, Salajeva 5, 41000 Zagreb, Croatia.
}

Manuscript received April 1, 1996; accepted September 6, 1996.

Coelho P. J., Duic N., Lemos C., Carvalho M. G., Aerospace Science and Technology, 1998, n ${ }^{\circ}$ 2, 107-1 19.

Abstract

Résumé
A computer code aimed at the simulation of the physical phenomena occuring in the solid fuel combustion chamber of a ramjet was extended to accommodate multi-block grids. The mathematical model is based on the numerical solution of the governing equations for mass, momentum, energy and transport equations for scalar quantities. The $k-\varepsilon$ model and the conserved scalar/prescribed probability density function formalism are employed. Heat and mass transfer at the walls are calculated using the wall functions of Chieng and Launder modified to account for porous walls. The multi-block method allows discontinuous grid lines along the interblock boundaries and transfer of data between neighbouring blocks satisfies flux conservation. The implementation of the multi-block domain decomposition technique is demonstrated for a turbulent isothermal flow through a pipe with a sudden expansion and for a solid fuel combustion chamber of a ramjet. In this case, the evolution of the local regression rate along the wall is in good qualitative agreement with the data, but quantitative disagreement was found, mainly due to the shortcomings of the turbulence model employed. (C) Elsevier, Paris

Keywords: Solid Fuel Combustion - Ramjets - Multi-block.

Modelage de la chambre de combustion d'un statoréacteur à combustible solide avec réseaux multiblocs. Un code de calcul destine à simuler les phénomènes physiques se déroulant dans une chambre de combustion de statoreacteur à combustible solide a été Ctendu pour prendre en compte les réseaux multiblocs. Le modele mathématique est base sur la resolution numérique des equations de bilan pour la masse, la quantité de mouvement et l'énergie ainsi que les equations de transport pour les quantités scalaires. Le modele $k$ - $\varepsilon$ et le formalisme du modele à scalaire passif et à fonction de densité de probabilité présumée sont employés. Les transferts de masse et de chaleur aux parois sont calculés en utilisant les fonctions de paroi de Chieng et Launder, modifiées pour tenir compte de la porosité. La méthode multiblocs que nous présentons autorise la discontinuité des lignes de maillage entre les blocs tout en préservant la conservativité des flux. La mise en œuvre de la technique de decomposition de domaines en blocs multiples s'effectue dans le cadre d'un Ccoulement turbulent isotherme. Deux configurations sont envisagées: un divergent d'une part, une chambre de combustion de statoreacteur à combustible solide d'autre part. Dans ce demier cas, l'évolution du taux de regression locale le long de la paroi est en bon accord qualitatif avec les données exptrimentales.Cependant, un d\&accord quantitatif, principalement dû aux limitations du modble de turbulence utilisé, a été observe. (C) Elsevier, Paris

Mots-cl\&s : Combustion de combustible solide Statoréacteur - Réseaux multiblocs. 


\section{NOMENCLATURE}

$\begin{array}{ll}a, b & \begin{array}{l}\text { Parameters of the probability density } \\ \text { function } \\ \text { Combined convection/diffusion coeffi- } \\ a_{i}\end{array} \\ b, c & \begin{array}{l}\text { cient } \\ \text { Coefficients of the linearized source term } \\ \text { of a discretized equation } \\ \text { Specific heat at constant pressure }\end{array} \\ C_{p} & \begin{array}{l}\text { Constant of the turbulence model } \\ C_{\mu}\end{array} C_{1}, C_{2}, C_{D} \\ D & \begin{array}{l}\text { Constants of the turbulence model } \\ \text { Diffusion conductance }\end{array}\end{array}$

E Constant in near-wall description of the velocity profile

$f \quad$ Weighting factor; mixture fraction

$F \quad$ Flux

$g \quad$ Gravitational acceleration

$h \quad$ Specific enthalpy

$h_{v} \quad$ Effective heat of gasification

$k \quad$ Turbulent kinetic energy

$M \quad$ Molar mass

$p \quad$ Pressure

$p(f) \quad$ Probability density function

$P \quad$ Jayatillaka function

$P e \quad$ Peclet number

$q \quad$ Heat flux

$r \quad$ Regression rate; radial coordinate

$R \quad$ Radius

$R_{0} \quad$ Universal gas constant

$t \quad$ Time

T Temperature

$u \quad$ Velocity component parallel to the wall

$u_{j} \quad$ Velocity component in $j$ direction

$U \quad$ Mean inlet velocity

$v_{w} \quad$ Velocity of the fuel blown through the wall

$x_{j} \quad$ Coordinate along $j$ direction

$y \quad$ Distance to the wall

$Y \quad$ Mass fraction

$\varepsilon \quad$ Dissipation rate of turbulent kinetic en-

ergy

$\kappa \quad$ Von Karman constant

$\mu \quad$ Dinamic viscosity

$\nu \quad$ Kinematic viscosity

$\rho \quad$ Density

$\sigma_{k}, \sigma_{\varepsilon} \quad$ Constants of the turbulence model

$\sigma_{t} \quad$ Constant of the turbulence model

$\phi \quad$ Arbitrary scalar variable

$\tau \quad$ Shear stress

\section{Subscripts}

$\begin{array}{ll}f_{u} & \text { Fuel } \\ N, S, E, W & \begin{array}{l}\text { North, south, east and west neighbouring } \\ \text { grid nodes of grid node } P\end{array} \\ n, s, e, w & \begin{array}{l}\text { North, south, east and west cell faces } \\ o\end{array} \\ \begin{array}{l}\text { Reference value } \\ \text { Grid node }\end{array} \\ t & \text { Turbulent } \\ v & \text { Viscous sublayer/fully turbulent region } \\ & \text { interface } \\ w & \text { Wall }\end{array}$

Superscripts

$\begin{array}{ll}(N) & \text { Block N } \\ (N B) & \text { Block NB } \\ o & \text { Standard state } \\ \left.()^{-}\right) & \text {Reynolds average } \\ (\sim) & \text { Favre average } \\ ()^{\prime \prime} & \text { Favre fluctuation }\end{array}$

\section{I - INTRODUCTION}

Nowadays, ramjets are drawing attention throughout the world because of their potential military and civilian applications. They can be used for the propulsion of missiles and projectiles, and for hypersonic or orbital aircrafts and space launchers. Compared to conventional rocket propulsion systems, ramjet propelled vehicles offer a considerable performance gain, because they use the oxygen in the atmosphere for combustion, instead of carrying the oxidizer. They have the potential to achieve an increased range and they offer increased effectiveness against maneuvering targets. The solid fuel ramjet is especially attractive for the propulsion of projectiles, because it is simple, cheap and has a high fuel density.

Figure 1 shows a sketch of a solid fuel ramjet combustor. It consists of an inlet, a solid fuel combustion chamber, and a nozzle. The incoming air is decelerated by shockwaves in the inlet to low subsonic velocities, yielding an increase of pressure and temperature. At the combustion chamber inlet, a diaphragm creates a sudden expansion of the incoming air, originating a recirculation zone required for flame stabilization. The solid fuel grain is a hollow cylinder which is pyrolyzed by the hot air. A gas phase diffusion flame resultant from the reaction between the fuel vapors and the air increases the temperature in the combustion chamber. In the nozzle, the thermal energy is converted to kinetic energy, yielding thrust.

The design of solid-fuel ramjet combustors has generally been based on empirical methods [1-2] and correlations [3-5], although more fundamental 


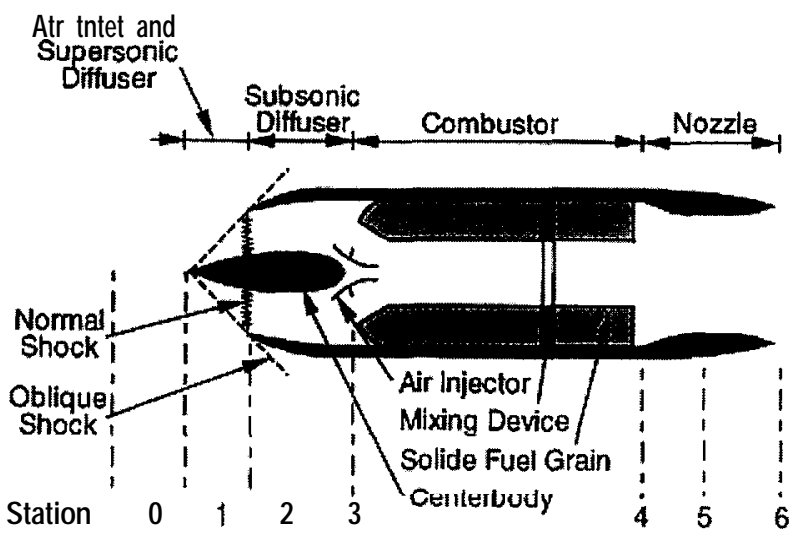

Fig. 1. - Sketch of a ramjet combustor.

mathematical models have also been developed [6-7]. At the TNO Prins Maurits Laboratory, in the Netherlands, the flow and combustion processes in a solid fuel combustion chamber have been studied both theoretically and experimentally [8-13]. A computer code, COPPEF, was developed to simulate the physical phenomena taking place in the combustion chamber [8-9]. This code was recently extended in order to handle multi-block grids and this extension is reported here. The main motivation behind this extension is the possibility to study in a more efficient way flows containing regions with very large gradients. In these regions, a very fine grid may be used, while outside these regions the grid may be much coarser.

The need for efficient flow solvers for engineering flow problems in complex geometries has motivated the development of several domain decomposition techniques, namely overlapping grids, multi-block grids and local grid refinement. Nowadays, multiblock techniques are extensively used in aeronautics to compute flows arround wings and aircrafts. A conservative treatment of interblock boundaries in the context of density-based flows has been developed by Rai [14], and many applications have been carried out since then. However, multi-block methods have received much less attention in the calculation of low Mach number flows using pressure correction solvers. The objective of this paper is to present a general multi-block domain decomposition technique and to demonstrate its application in the mathematical modelling of the solid fuel combustion chamber of a ramjet. Although the method is presented here for Cartesian/cylindrical coordinates, its extension to curvilinear coordinates is straightforward.

In the next section, the computational model describing the flow and combustion processes will be presented focusing on the calculation of the heat transfer and the solid fuel regression rate at the solid fuel wall. The multi-block domain decomposition technique is described next. Then, the results obtained are presented and discussed. Conclusions are drawn in the last section.

\section{II - THE MATHEMATICAL MODEL}

In the present work the COPPEF computer code developed by Vos [8-9] and recently extended to accommodate a multi-block grid was used. This code is aimed at the calculation of turbulent flows in twodimensional Cartesian or axisymmetric geometries, and it includes subroutines suitable for the simulation of a solid fuel combustion chamber of a ramjet. Before addressing the extension to multi-block grids, a brief overview of the physical models is given. Further details may be found elsewhere [8-9].

The mathematical model is based on the solution of the Favre-averaged conservation equations for mass, momentum, energy and transport equations for scalar quantities. In turbulent flows with high Reynolds numbers these equations may be written in Cartesian coordinates as

$$
\begin{gathered}
\frac{\partial \bar{\rho}}{\partial t}+\frac{\partial}{\partial x_{j}}\left(\rho \tilde{u}_{j}\right)=0 \\
\frac{\partial}{\partial t}\left(\bar{\rho} \tilde{u}_{i}\right)+\frac{\partial}{\partial x_{j}}\left(\bar{\rho} \tilde{u}_{i} \tilde{u}_{j}\right) \\
=-\frac{\partial}{\partial x_{j}}\left(\bar{\rho} \tilde{u}_{i}^{\prime \prime} u_{j}^{\prime \prime}\right)-\frac{\partial \bar{p}}{\partial x_{i}}+\bar{\rho} g_{i}, \\
\frac{\partial}{\partial t}\left[\bar{\rho}\left(\bar{h}+\frac{1}{2} \tilde{u}_{i}^{2}+k\right)\right] \\
+\frac{\partial}{\partial x_{j}}\left[\rho u_{j}\left(\tilde{h}+\frac{1}{2} \tilde{u}_{i}^{2}+k\right)\right] \\
-\frac{\partial \bar{p}}{\partial t}=\bar{\rho} \tilde{u}_{i} g_{i}-\frac{\partial}{\partial x_{j}}\left(\bar{\rho} \widetilde{u}_{i}^{\prime \prime} u_{j}^{\prime \prime} \tilde{u}_{i}\right) \\
-\frac{\partial}{\partial x_{j}}\left(\bar{\rho} \widetilde{u}_{j}^{\prime \prime} k^{\prime \prime}+\bar{\rho} \widehat{u}_{j}^{\widehat{\prime \prime} h^{\prime \prime}}\right)-\frac{\partial}{\partial x_{j}} \bar{q}_{j}, \\
\frac{\partial}{\partial t}(\bar{\rho} \tilde{\phi})+\frac{\partial}{\partial x_{j}}\left(\bar{\rho} \tilde{u}_{j} \tilde{\phi}\right) \\
=-\frac{\partial}{\partial x_{j}}\left(\bar{\rho} \widetilde{u}_{j}^{\prime \prime} \phi^{\prime \prime}\right)+\bar{S}_{\phi} .
\end{gathered}
$$

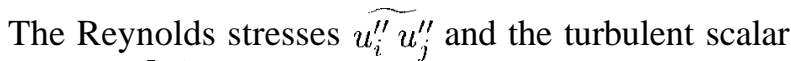
fluxes $\widetilde{u_{i}^{\prime \prime} \phi^{\prime \prime}}$ are estimated using the $k-\varepsilon$ eddy viscosity/diffusivity turbulence model:

$$
\begin{aligned}
& -\bar{\rho} \widetilde{u_{i}^{\prime \prime} u_{j}^{\prime \prime}}=\mu_{t}\left(\frac{\partial \tilde{u}_{i}}{\partial x_{j}}+\frac{\partial \tilde{u}_{j}}{\partial x_{i}}\right) \\
& -\frac{2}{3}\left(\rho k+\mu_{t} \frac{\partial \tilde{u}_{k}}{\partial x_{k}}\right) \delta_{i j},
\end{aligned}
$$




$$
-\bar{\rho} \widetilde{u_{j}^{\prime \prime} \phi^{\prime \prime}}=\frac{\tilde{\mu}_{t}}{\sigma_{t}} \frac{\partial \tilde{\phi}}{\partial x_{j}}
$$

and

$$
\mu_{t}=C_{\mu} \bar{\rho} k^{2} / \varepsilon .
$$

This model involves the solution of transport equations for the turbulent kinetic energy, $k$, and its dissipation rate, $\varepsilon$ :

$$
\begin{gathered}
\frac{\partial}{\partial t}(\bar{\rho} k)+\frac{\partial}{\partial x_{j}}\left(\bar{\rho} \tilde{u}_{j} k\right) \\
=\frac{\partial}{\partial x_{j}}\left(\frac{\mu_{t}}{\sigma_{k}} \frac{\partial k}{\partial x_{j}}\right)-\bar{\rho} \widetilde{u_{i}^{\prime \prime} u_{j}^{\prime \prime}} \frac{\partial \tilde{u}_{i}}{\partial x_{j}} \\
-\frac{\mu_{t}}{\rho^{2} \sigma_{t}} \frac{\partial \bar{\rho}}{\partial x_{j}} \frac{\partial \bar{p}}{\partial x_{j}}-\bar{\rho} \varepsilon_{;} \\
\frac{\partial}{\partial t}(\bar{\rho} \varepsilon)+\frac{\partial}{\partial x_{j}}\left(\bar{\rho} \tilde{u}_{j} \varepsilon\right) \\
=\frac{\partial}{\partial x_{j}}\left(\frac{\mu_{t}}{\sigma_{\varepsilon}} \frac{\partial \varepsilon}{\partial x_{j}}\right) \\
+C_{1} \frac{\varepsilon}{k}\left(-\bar{\rho} \widehat{u_{j}^{\prime \prime} u_{j}^{\prime \prime}} \frac{\partial \tilde{u}_{i}}{\partial x_{j}}-\frac{\mu_{t}}{\rho^{2} \sigma_{t}} \frac{\partial \bar{\rho}}{\partial x_{j}} \frac{\partial \bar{p}}{d x j}\right) \\
-C_{2} \bar{\rho} \frac{\varepsilon^{2}}{k} .
\end{gathered}
$$

The constants of the model are $\mathrm{C},=0.09, C_{1}=1.44$, $C_{2}=1.92, \sigma_{k}=1.0, \sigma_{\varepsilon}=1.3$ and $\sigma_{t}=0.7$.

The enthalpy of the reactive mixture is defined as

$$
h=\sum_{j} Y_{j}\left(h_{j}^{0}+\int_{T_{0}}^{T} C_{p, j}(T) d T\right),
$$

where index $j$ runs over all the chemical species, and the equation of state is written as

$$
p=\rho R_{0} T \sum\left(Y_{j} / M_{j}\right)
$$

Combustion is modelled using the conserved scalar/prescribed probability density function (p.d.f.) formalism. It is assumed that combustion is described by a single one-step irreversible reaction between the fuel and the oxidizer yielding combustion products. This assumption provides relationships between instantaneous values of the chemical species concentrations and the conserved scalar, taken as the mixture fraction.

The mean values of the chemical species concentrations, temperature and density may be found from integration of the product of the instantaneous value by the p.d.f. over the mixture fraction range

$$
\tilde{\phi}={ }_{\mathrm{I}_{0}}^{.1} \phi(f) p(f) d f .
$$

$$
\begin{gathered}
\bar{\phi}=\bar{\rho} \int_{0}^{1} \frac{\phi(f)}{\rho(f)} p(f) d f, \\
\bar{\rho}=\left[\int_{0}^{1} \frac{p(f)}{\rho(f)} d f\right]^{-1} .
\end{gathered}
$$

The beta p.d.f. was chosen. It is completely defined in terms of the mean value, $\tilde{f}$, and variance, $\tilde{f}^{\prime \prime 2}$, of the mixture fraction:

$$
p(f)=\frac{f^{a-1}(1-f)^{b-1}}{\int_{0}^{1} f^{a-1}(1-f)^{b-1} d f} .
$$

where

$$
\begin{gathered}
a=\tilde{f}\left(\tilde{f}(1-\tilde{f}) / \tilde{f}^{\prime \prime 2}-1\right), \\
b=(1-\tilde{f}) a / \tilde{f} .
\end{gathered}
$$

Transport equations are solved to compute $\tilde{f}$ and $\tilde{f}^{\prime \prime 2}$

$$
\begin{aligned}
& \frac{\partial}{\partial t}(\bar{\rho} \tilde{f})+\frac{\partial}{\partial x_{j}}\left(\bar{\rho} \tilde{u}_{j} \tilde{f}\right)=\frac{\partial}{\partial x_{3}}\left(\frac{\mu_{t}}{\sigma_{p}} \frac{\partial \tilde{f}}{\partial x_{i j}}\right) \\
& \frac{\partial}{\partial t}\left(\bar{\rho} \tilde{f}^{\prime \prime 2}\right)+\frac{\partial}{\partial x_{j}}\left(\bar{\rho} \tilde{u}_{j} \tilde{f}^{\prime \prime 2}\right)=\frac{\partial}{\partial x_{j}}\left(\frac{\mu_{t}}{\sigma_{t}} \frac{\partial \tilde{f}^{\prime \prime 2}}{\partial x_{j}}\right) \\
& \quad+\frac{2 \mu_{t}}{\sigma_{t}}\left(\frac{\partial \tilde{f}}{\partial x_{j}}\right)^{2}-C_{D} \frac{\bar{\rho} \varepsilon}{k} \tilde{f}^{\prime \prime 2}
\end{aligned}
$$

where $C_{D}$ has a value of 2.0.

A finite rate chemical kinetics combustion model is also available in the code. However, it does not account for the turbulence/combustion interaction and, therefore, it was not used in the present calculations.

The $k \quad \varepsilon$ turbulence model is not valid close to the walls, where the Reynolds number is low. In such regions, the wall functions of Chieng and Launder [ 15], modified to account for porous walls with blowing or suction, were employed. These wall functions play a crucial role in the determination of the regression rate, a very important parameter in the analysis of solid fuel combustion chambers. The calculation of this rate is explained below.

The wall function method of Chieng and Launder assumes that the boundary layer near a solid wall can be divided into two regions, a viscous sublayer, in the immediate vicinity of the wall, and a fully turbulent region. The interface between the two regions is located at $\mathrm{Re},=20 \mathrm{.} \mathrm{Re}$, is the Reynolds number based on the distance to the wall, $y_{v}$, and on the local square root of the turbulent kinetic energy, $k$, ,. The turbulent kinetic energy is assumed to have a parabolic distribution in the viscous sublayer and a linear distribution in the fully turbulent region. This assumed distribution allows the calculation of $y_{v}$ and $k$, at the interface. 
In the fully turbulent region, the velocity component parallel to the wall, estimated from a Couette flow analysis, is given by [ 16].

$$
\begin{aligned}
u= & \frac{\tau_{w} / \rho}{\kappa C_{\mu}^{1 / 4} k_{v}^{1 / 2}} \ln \left(E y C_{\mu}^{1 / 4} k_{v}^{1 / 2} / \nu\right) \\
& +v_{u}\left(\frac{\ln \left(E y C_{\mu}^{1 / 4} k_{v}^{1 / 2} / \nu\right)}{2 \kappa}\right)^{2}
\end{aligned}
$$

where the second term accounts for blowing or suction effects. In this case, $E$ is no longer a constant but depends on the local flow conditions. Equation (19) is valid in the $\log$ layer which typically lies between $y^{+}=30$ and $y^{+}=0.1 \delta$, where $y^{+}$is the dimensionless distance from the wall and $\delta$ is the boundary layer thickness. In the viscous sublayer, which extends from the wall up to $y^{+}=5$, the shear stress distribution is given by:

$$
\tau=\tau_{w}+\rho v_{w} u=\mu \frac{d u}{d y} \cong \mu \frac{u}{y} .
$$

Applying equation (19) to the grid node close to the wall, and applying equations (19) and (20) to the interface between the viscous sublayer and the fully turbulent region, a system of three equations is obtained from which $\tau_{w}, u_{v}$ and $E$ may be calculated.

A Couette flow analysis also shows that the heat flux to the wall may be calculated as:

$$
\begin{aligned}
& \frac{\rho C_{p}\left(T_{w}-T\right) k_{v}^{1 / 2} C_{\mu}^{1 / 4}}{q_{w}} \\
& =\left[\left(1+\frac{v_{w} u_{P}}{\tau_{w} / \rho}\right)^{\sigma_{t}} \exp \left(\frac{\sigma_{t} v_{w} P}{k_{v}^{1 / 2} C_{\mu}^{1 / 4}}\right)-1\right] \\
& /\left(v_{w} /\left(k_{v}^{1 / 2} C_{\mu}^{1 / 4}\right)\right) .
\end{aligned}
$$

The range of validity of this equation is identical to that of equation (19). The heat flux to the wall, $4 w$, may be related to the velocity of the fuel blown through the wall into the flow, v,.

$$
q_{w}=h_{v} \rho_{f u} v_{w}
$$

where $\rho_{f u}$ is the density of the gaseous fuel and $h_{v}$ is the effective heat of gasification, i.e., the amount of heat required to pyrolyze $1 \mathrm{~kg}$ of fuel. The temperature of the wall, $T_{w}$, is given as a boundary condition, and equations (21) and (22) are used to compute $q_{w}$ and $v_{w}$. The local regression rate of the fuel, $r$, is then calculated from:

$$
r=q_{w} /\left(\rho_{w} h_{v}\right)
$$

where $\rho_{w}$ is the density of the solid fuel.

The governing equations are integrated in each control volume using a finite-volume method and a multi-block domain decomposition technique, which is described in the next section. The equations are discretized using central differences (second-order accuracy) for all but the convective terms which are discretized using the power-law scheme (order of accuracy between 1 and 2). The unsteady terms are discretized using the fully implicit method. However, only steady-state problems are dealt with in the present work. In this case, the discretized equations may be written in the following general form (see Patankar [17] for details):

$$
a_{P} \phi_{P}=\sum a_{i} \phi_{i}+b
$$

where index $i$ runs over all the neighbouring grid nodes of the central node $P$. The combined convectivediffusive coefficients $a_{i}$ are calculated according to the power-law scheme as:

$$
\begin{array}{r}
a_{N}=D_{n} \max \left[\begin{array}{ll}
0,(1 & 0.1|P e|_{n}
\end{array}\right)^{5}+\max \left[-F_{n}, 0\right] \\
a_{S}=D_{s} \max \left[0,\left(\begin{array}{ll}
1 & 0.1|P e|_{s}
\end{array}\right)^{5}+\max \left[F_{s}, 0\right]\right.
\end{array}
$$

and similarly for $a_{E}$ and $a_{W}$, and the coefficient of $\phi_{P}$ is given by

$$
a_{P}=\sum a_{i}-c
$$

In equations (24) and (26) b and c are the coefficients of the linearized source term, $S_{\phi}$, of variable $\phi: S_{\phi}=$ $b+\mathrm{c} \phi_{P}$. In equation (25) $F$ denotes the convective mass flux per unit area and $D$ represents the diffusion conductance, defined as the ratio between the diffusion coefficient and the distance between neighbouring grid nodes. The ratio $F / D$ is the Peclet number, Pe.

A staggered grid variable arrangement is employed. Pressure-velocity coupling is accomplished by means of the SIMPLE, SIMPLEC or SIMPLER algorithms. Due to the iterative nature of the solution algorithm, conservation of mass is not satisfied at the control volume level during the course of the iterative process. To enforce overall continuity, an overall line continuity correction method is also incorporated. This method corrects the velocity field in streamwise direction and the pressure field in order to satisfy overall continuity at every grid line in the crosswise direction [8]. The solution of the systems of discretized equations is carried out using a segregated solution approach by means of the Gauss-Seidel line-by-line iterative procedure.

\section{III - THE MULTI-BLOCK DOMAIN DECOMPOSITION METHOD}

In the multi-block method the physical domain is divided into several subdomains (blocks), and a grid is 
generated for each block separately. In this way, fine grids need only be used in regions of steep gradients, whereas coarse grids may be employed in regions of smooth variation of the dependent variables. To solve the governing equations in such a domain, information must periodically be exchanged among the blocks. In every iteration of the solution algorithm all the blocks are treated in sequence, and at the beginning of the calculations in a block the required data from the neighbouring books is transferred to the block under consideration. This exchange of information is a key issue of a multi-block solver.

A staggered grid system is used in the COPPEF code. To allow for the exchange of information mentioned above, neighbouring blocks overlap at the interblock boundaries in such a way that, for each block, one line of grid nodes is placed beyond the interblock boundary (e.g., grid line $j=J M A X-1$ in block $\mathrm{N}$ shown in Figure 2). In the original version of the COPPEF code one line of grid nodes was already placed outside of the computational domain to simplify the boundary conditions treatment. This means that the computational domain ranged from $i=2$ to $i=I M A X-1$, and from $j=2$ to $j=J M A X-1$, the lines $i=1, i=I M A X, j=1$ and $j=\mathbf{J}$ MAX being used to handle the boundary conditions. This structure was kept unchanged in the new multi-block version of the code. Hence, a second grid line is placed beyond the interblock boundary (e.g., $j=$ J MAX is block $\mathbf{N}$ of Figure 2). This second grid line is not needed for the zonal treatment, but it allows the use of the same grid structure in all the blocks, regardless of their location.

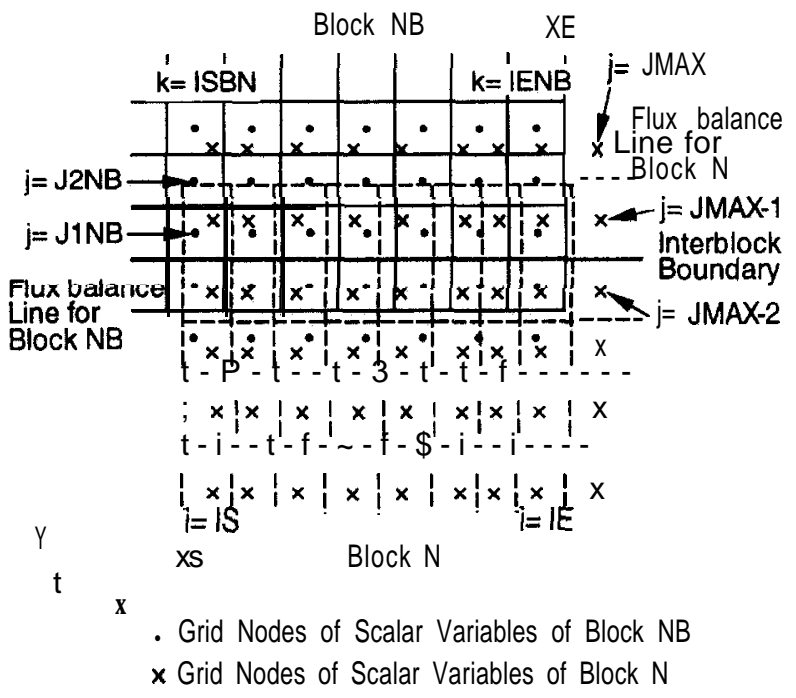

Fig. 2. - Grid detail to illustrate the calculation of the fluxes.

In the multi-block method, each block is treated sequentially in each iteration. Solution of the governing equations in a given block requires the specification of the boundary conditions for that block.
Part of the block boundary does not coincide with the boundary of the physical domain but it is shared with one or more other blocks. The identification of the kind of boundary for each grid node along the boundary, as well as the identification of the neighbouring block in the case of an interblock boundary, is performed prior to the beginning of the iterative procedure and stored in an array.

One of the key features of the finite volume method is that it naturally ensures conservation of fluxes. Although conservation is easy to ensure if a single-block grid is considered, care must be taken when computing the fluxes across the interblock boundaries for multi-block grids, if conservation is to be maintained. To ensure conservation, the fluxes must be identical when estimated from the blocks on either side of an interblock boundary.

To explain the calculation of fluxes of scalar variables at the interblock boundaries, the example illustrated in Figure 2 is considered. Let $\mathrm{N}$ be the block to be treated and $N B$ the neighbouring block. Suppose that IS and $I E$ are the limits, in block N, of the interblock boundary between blocks N and NB. The south boundary of block NB is swept to identify the indices of the grid nodes of that block that share the interblock boundary with block $\mathrm{N}$. Let ISNB and $I E N B$ be the lower and upper limits of the interblock boundary in block NB as shown in Figure 2.

The governing equations are solved for block $\mathrm{N}$ up to $j=J \mathbf{M A X} 1$ (see Fig. 2). Thus, a boundary condition involving data from block NB is required for the north face of the control volumes of block $\mathrm{N}$ with $j=J$ MAX -1 . The line containing the north faces of such control volumes is referred to as the flux balance line for block N. A flux balance line for block NB may be defined similarly. The region between the two flux balance lines is the overlapping region.

Global flux conservation across the flux balance line requires that the following identity is satisfied

$$
\int_{X S}^{X E} F^{(N)}(x) d x=\int_{X S}^{X E} F^{(N B)}(x) d x .
$$

where $X S$ and $\mathbf{X E}$ are the $x$ limits of the interblock boundary between blocks N and NB, as shown in Figure 2. $F^{(N)}$ and $F^{(N B)}$ are the fluxes (convective plus diffusive) computed for blocks $\mathrm{N}$ and NB, respectively.

Local flux conservation across the flux balance line requires that for every control volume of block $\mathrm{N}$ with indices $i$ such that $\mathbf{I S} \leq i \leq \mathbf{I E}$, and $j=J \mathbf{M} \mathbf{A X}-1$ the following relation holds:

$$
\int_{x_{i-1 / 2}^{(N)}}^{x_{i+1 / 2}^{(N)}} F^{(N)}(x) d x=\int_{x_{i-1 / 2}^{(N)}}^{x_{i+1 / 2}^{(N)}} F^{(N B)}(x) d x,
$$

where $x_{i+1 / 2}^{(N)}$ and $x_{i-1 / 2}^{(N)}$ are the $x$ coordinates of the cell faces of control volumes of block $\mathrm{N}$ with 
index $i$. Local flux conservation implies global flux conservation, i.e., satisfaction of equation (28) for all control volumes of block $\mathrm{N}$ such that IS $<i<I E$ and $j=J M A X-1$ automatically ensures that equation (27) is also satisfied.

Assuming a piecewise constant variation of $F^{(N B)}$, discretization of equation (28) yields:

$$
F_{i}^{(N)}-\sum_{k=I S N B}^{I E N B} f_{i, k} F_{k}^{(N B)},
$$

where $f_{i, k}$ is a weighting factor equal to the fraction of the area of the south face of control volume $(k, J 1 \mathrm{NB})$ of block $N B$ that shares the north face of control volume ( $i, J M A X-2)$ of block $\mathrm{N}$.

The flux computation along the interblock boundary under consideration requires a sweep in block $\mathrm{N}$ from $i=\mathrm{IS}$ to $i=I E$ and, for each $(i, J M A X-1)$ cell, involves the calculation of the flux $F_{i}^{(N)}$ according to equation (29). Other interblock boundaries are treated likewise.

The flux $F_{k}^{(N B)}$ of a scalar variable $\phi$ across the flux balance line is calculated as follows:

$$
F_{k}^{(N B)}=a_{N_{k}, J 1 N B}^{(N B)}\left(\phi_{k, J 2 N B}^{(N B)}-\phi_{k, J 1 N B}^{(N B)}\right) .
$$

The combined convection/diffusion coefficient, $a_{N}$, is determined from the mass flow rate and the diffusion flux (Eq. 25a), both evaluated at the flux balance line using the data of block NB. Hence, the fluxes at the flux balance lines are determined in the same way as the fluxes at cell faces in the interior of the domain. Therefore, the multi-block interface treatment does not deteriorate the general spatial accuracy of the method. The discretized equation of the scalar variable $\phi$ at a grid node $P$ (indices $i, j$, with $I S<i \leq I E$ and $j=J M A X-1)$ of block $\mathrm{N}$ is obtained from equations (24), (29) and (30) yielding

$$
\begin{aligned}
& \left(a_{S_{i, J M A X-1}}^{(N)}+a_{E_{i, J M A X-1}}^{(N)}+a_{W_{i, J M A X-1}}^{(N)}-c\right) \\
& \quad \times \phi_{P}^{(N)}=a_{S_{i, J M A X-1}}^{(N)} \phi_{S}^{(N)}+a_{E_{i, J M A X-1}^{(N)}}^{(N)} \phi_{E}^{(N)} \\
& +a_{W_{i, J M A X-1}}^{(N)} \phi_{W}^{(N)}+\sum_{k=I S N B}^{I E N B} f_{i, k} a_{N_{k, J 1 N B}}^{(N B)} \\
& \quad \times\left(\phi_{k, J 2 N B}^{(N B)}-\phi_{k, J 1 N B}^{(N B)}\right)+b .
\end{aligned}
$$

The treatment of the u-velocity component along horizontal interblock boundaries and of the v-velocity component along vertical interblock boundaries are similar to that described above. The differences resultant from grid staggering do not present any difficulties. The IL velocity component, calculated from the convective flux at vertical flux balance lines, is used as a boundary condition for the u-momentum equation along vertical boundaries. Similarly, the $v$ velocity component, calculated from the convective flux at horizontal flux balances lines, is used as a boundary condition for the v-momentum equation along horizontal boundaries.

The treatment described above does not apply to the pressure correction equation. In this case, the mass flow rate across an interblock boundary is calculated as in the other equations. The coefficient of the discretized equation for the cell face lying on the interblock boundary is set equal to zero and the mass flow rate is directly inserted into the source term.

In the case of isothermal flows, the governing equations only involve pressure gradients. Hence, if a multi-block grid is employed, independent pressure fields may develop within each block, even if the pressure is prescribed at a certain location. In fact, due to the arrangement of staggered grid variables, the pressure within a block is not required to solve the equations in the neighbouring blocks. Although this development of independent pressure fields does not prevent achieving convergence, it is not desirable whenever knowledge of the pressure field is important for the problem under consideration.

In the case of reactive flows, the situation is more complex because the pressure field is used to compute the temperature by means of the equation of state. Therefore, if independent pressure fields were obtained in different blocks convergence could not be achieved. Hence, we have devised a simple method to adjust the pressure within the blocks, in such a way that the global pressure within the grid is identical to the one predicted using a single-block grid. The method consists of the selection of an interblock boundary for each block, calculation of the total pressure force along that interblock boundary for one of the blocks, and adjustment of the pressure field in the other block, such that the total pressure force along the interblock boundary computed in each one of the two blocks is the same.

The multi-block strategy outlined above was incorporated in the COPPEF code. To improve convergence, global as well as block mass conservation was enforced during the course of the iterative procedure.

Extension of the present multi-block method to three-dimensional geometries does not present any difficulties: equations (27) and (28) would involve double-integrals and equation (29) would involve a double-summation, extending over the twodimensional flux balance surfaces. The extension to curvilinear grids implies that equations (27) and (28) are established for a curvilinear flux balance line, i.e., $x$ is replaced by the curvilinear coordinate. Moreover, the flux balance line for block $\mathrm{N}$ does not necessarily lie between two $j=$ constant grid lines of block $N B$. But at an interblock boundary, the flux at the north face of a control volume of block $\mathrm{N}$, for example, is still obtained adding the weighted contributions calculated using the data from block $N B$. The key ideas outlined above for Cartesian/cylindrical coordinates 
remain unchanged. Details on the implementation of a similar interblock boundary treatment applied to curvilinear grids may be found in Shyy et al. [ 18].

\section{IV - RESULTS AND DISCUSSION}

Calculations are firstly presented for a turbulent isothermal flow, for validation purposes. Then, an application to a solid fuel combustion chamber of a ramjet is given. In this case, available data is restricted to the local mean regression rate.

\section{IV.1 - Turbulent flow through a sudden pipe expansion}

A turbulent isothermal flow of air through a pipe with a sudden expression at the inlet was considered for validation purposes. The length of the pipe is $1 \mathrm{~m}$, the diameter downstream of the expansion is $0.2 \mathrm{~m}$ and the inlet diameter is $0.1 \mathrm{~m}$. Calculations were performed for a Reynolds number based on the inlet diameter equal to $4 \times 10$ ".
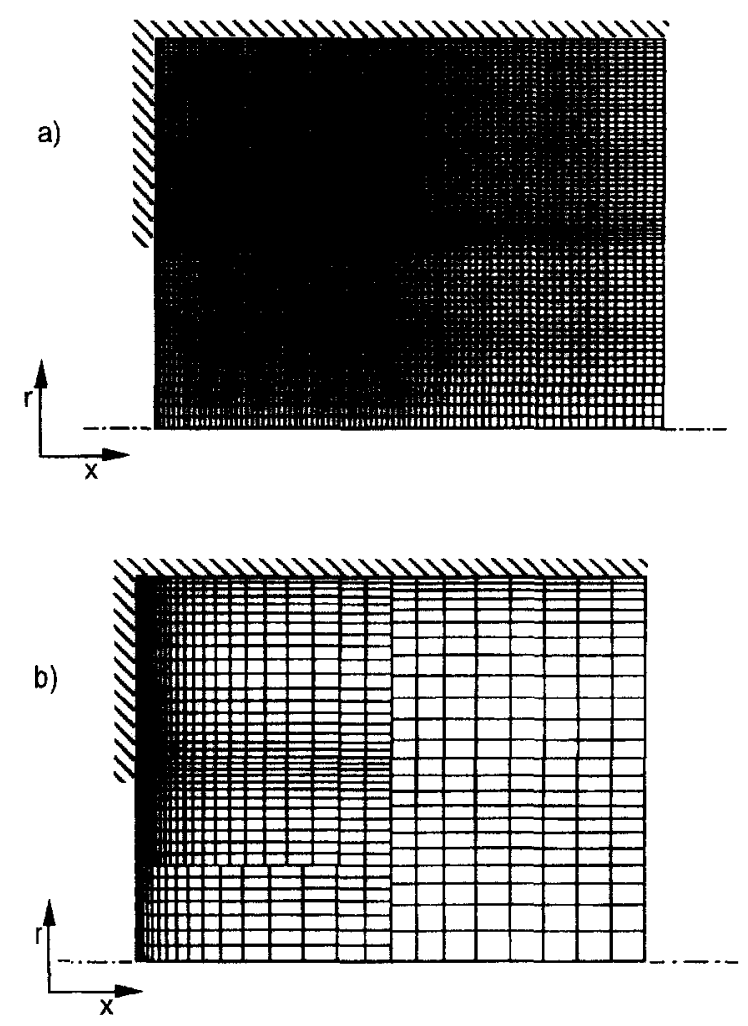

Fig. 3. - Grids used to compute the turbulent flow through a sudden pipe expansion. a) Single-block grid ; b) Multi-block grid.

The results presented here were obtained using a non-uniform single-block grid with $80 \times 60$ grid nodes (see Fig. 3 a) and a three-block grid with discontinuous grid lines along the interblock boundaries (see
Fig. 3 b). The aspect ratio of the grids plotted in Figure 3 is different from the actual one, i.e., the radial dimension was increased to avoid clustering of the horizontal grid lines. In the multi-block case, grid lines were concentrated on the recirculating and reattaching regions and fewer grid lines were placed elsewhere.

In order to check the correctness of the exchange of data between neighbouring blocks, additional calculations were carried out using a three-block grid with continuous grid lines along the interblock boundaries and with grid nodes coincident with those of the single-block grid. It was found that the predictions obtained in this case are similar to the single-block predictions, as expected. Therefore, the multi-block calculations with continuous grid lines along the interblock boundaries are not shown here. They have required about $10 \%$ more iterations than the corresponding single-block calculations. This increase of the number of iterations is partly due to the interblock boundaries, which require an explicit treatment of the fluxes across them, and partly due to the different underrelaxation parameters employed, as explained below.

In the case of multi-block calculations it was found that during the first few iterations it is necessary to use small underrelaxation factors for $k$ and $\varepsilon$. In fact, during the first few iterations, large changes in the dependent variables occur from iteration to iteration, unless the initial guess is close to the converged solution. During iteration number $n$, when block number $k$ is treated, $n$ iterations have already been performed for blocks 1 to $k-1$, whereas only $n-1$ iterations have been peformed for the remaining blocks. Since in every iteration the blocks are always swept in the same order, this means that the interblock boundary data is obtained from the values of the dependent variables calculated in the current iteration (n) for blocks 1 to $k-1$, and from the values of the previous iteration $(n-1)$ for the remaining blocks. This causes large discontinuities in the pressure and velocity fields along the interblock boundaries at the beginning of the iterative procedure. These discontinuities induce high levels of turbulent kinetic energy and dissipation rate yielding divergence of the iterative procedure. To prevent the appearance of these unphysical high levels of $k$ and $\varepsilon$, it is necessary to use small underrelaxation factors until a relatively stabilized velocity field has been established and unphysical gradients along the interblock boundaries have been eliminated. Hence, $k$ and $\varepsilon$ underrelaxation factors one order of magnitude smaller than their final values were used up to iteration number 30 . Thereafter, they were multiplied by a factor 10 and kept unchanged up to the end of the convergence process.

The predicted u-velocity contours of the single and multi-block grids are shown in Figures $4 \mathrm{a}$ and 4 b, respectively. The three-block grid with discontinuous grids lines along the interblock boundaries yields 


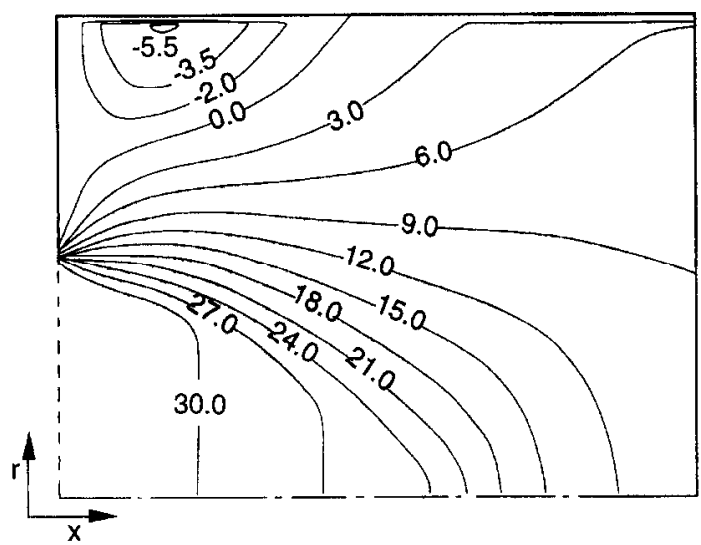

a

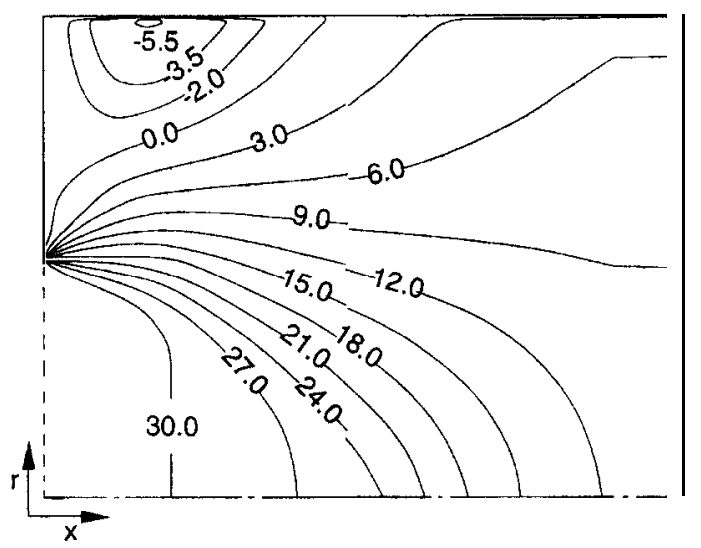

b

Fig. 4. Predicted $u$-velocity $(\mathrm{m} / \mathrm{s})$ contours for the flow through a sudden pipe expansion. a) Single-block grid ; b) Multi-block grid.

predicted u-velocity contours with none or very small discontinuities at the interblock boundaries. Moreover, the slope of the contours is also continuous across the interblock boundaries. This demonstrates the quality of the method. Small discontinuities occur in regions where the grid is fine on one side of the interblock boundary and coarse on the opposite side (see Fig. 3 b). They are due to the plotting routine which determines the contours using linear interpolations of the data computed for each block.

Figure 5 shows radial profiles of the u-velocity component at four different axial stations together with the experimental data [19]. The u-velocity, normalized by the mean inlet velocity, is plotted against the radial coordinate, normalized by the radius of the pipe downstream of the expansion. The profiles computed using the two different grids are barely distinguishable. No discontinuities are found across the horizontal interblock boundary, showing the quality of the interblock boundary treatment.

The predictions are fairly good in the reattachment region, but the discrepancies between the predictions and the measurements increase farther downstream. A similar behaviour was observed by Vos and van Dijk [20]. The slow redevelopment of the flow downstream of the reattachment point has also been predicted by the $k-\varepsilon$ model in other flows, such as the backward facing step flow and the flow over a square obstacle [21]. It is related to the shortcomings of the turbulence model.

An important parameter in the analysis of the flow in a sudden pipe expansion is the reattachment length. The experimentally observed reattachment length, normalized by the step height (difference between the radius downstream and the radius upstream of the expansion), is in the range 8.5-9 [19, 22, 23$]$. In the calculations reported here, the normalized reattachment length is 8.86 and 9.30 for the single and multi-block grids, respectively. These values are in close agreement with the experimentally observed range, and with other predictions reported in the literature for the same geometry [23-25]. Therefore, the $k-\varepsilon$ model predicts the reattachment length within the experimental uncertainty, in contrast to the predictions of the backward facing step flow, as discussed by Nallasamy [21]. The predicted maximum reverse velocity is similar for the two grids considered: 0.181 for the- single-block grid and 0.182 for the multi-block grid.

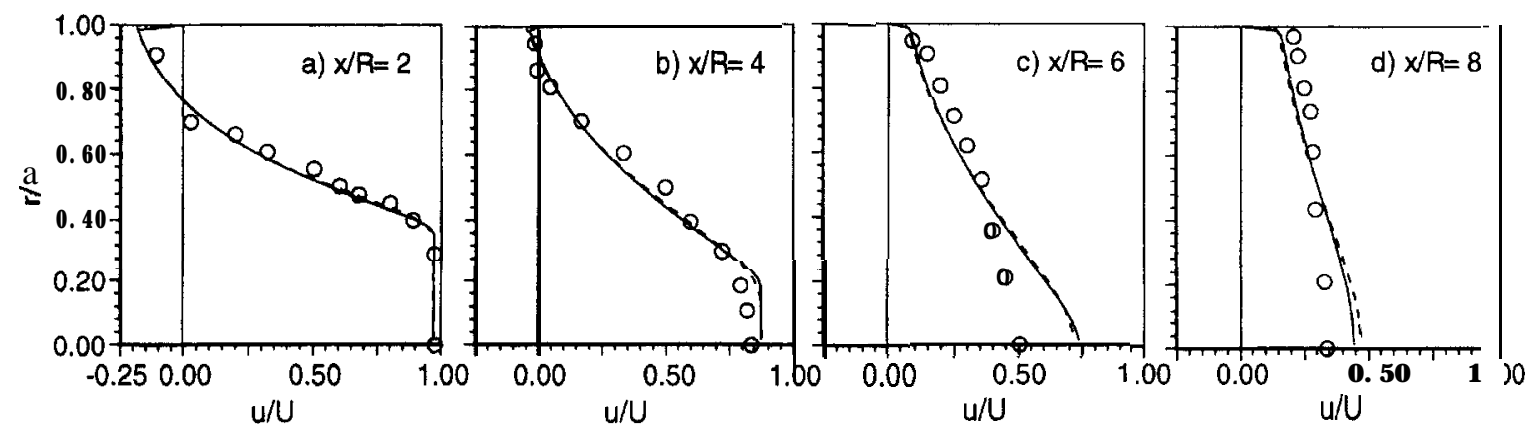

Fig. 5. - Predicted and measured radial u-velocity profiles for the flow through a sudden pipe expansion (symbols: measurements [16], solid line: single-block grid, dashed line: multi-block grid). 

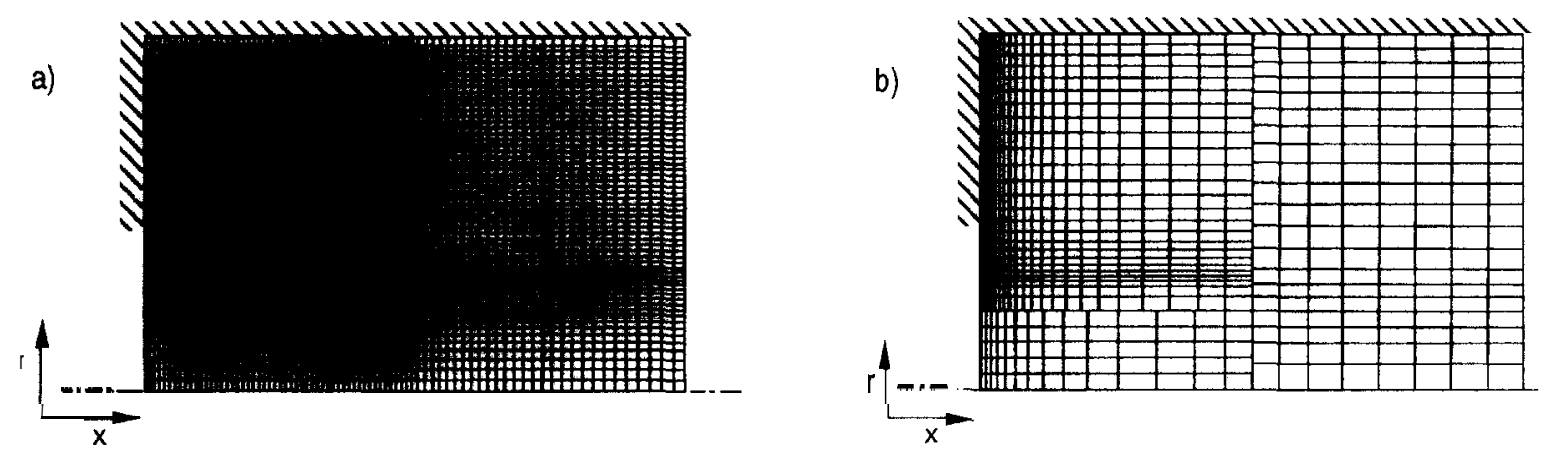

Fig. 6. - Grids used to compute the flow in a solid fuel combustion chamber. a) Single-block grid : b) Multi-block grid

\section{IV.2 - Reactive flow in a solid fuel combustion chamber of a ramjet}

Following the evaluation of the domain decomposition technique for a turbulent isothermal flow described above, the code was applied to a solid fuel combustion chamber of a ramjet for which experimental data is available [ 13]. The combustor chamber, schematically shown in Figure 1, has a length of $300 \mathrm{~mm}$. The inner diameter of the combustion chamber increases along the time due to fuel (polyethylene) pyrolysis, but this process is not taken into account by the mathematical model. The initial fuel grain diameter is $40 \mathrm{~mm}$ but, since the experimental mean regression rate is about $0.2 \mathrm{~mm} / \mathrm{s}$, the fuel grain diameter was set equal to $45 \mathrm{~mm}$ for calculation purposes. This is an average value of the inner diameter during combustion for the measurements carried out. Air at a temperature of $300 \mathrm{~K}$ and a pressure of $0.40 \mathrm{MPa}$ is admitted into the combustion chamber through a port of $15 \mathrm{~mm}$ diameter. The air mass flow rate is $150 \mathrm{~g} / \mathrm{s}$. a)
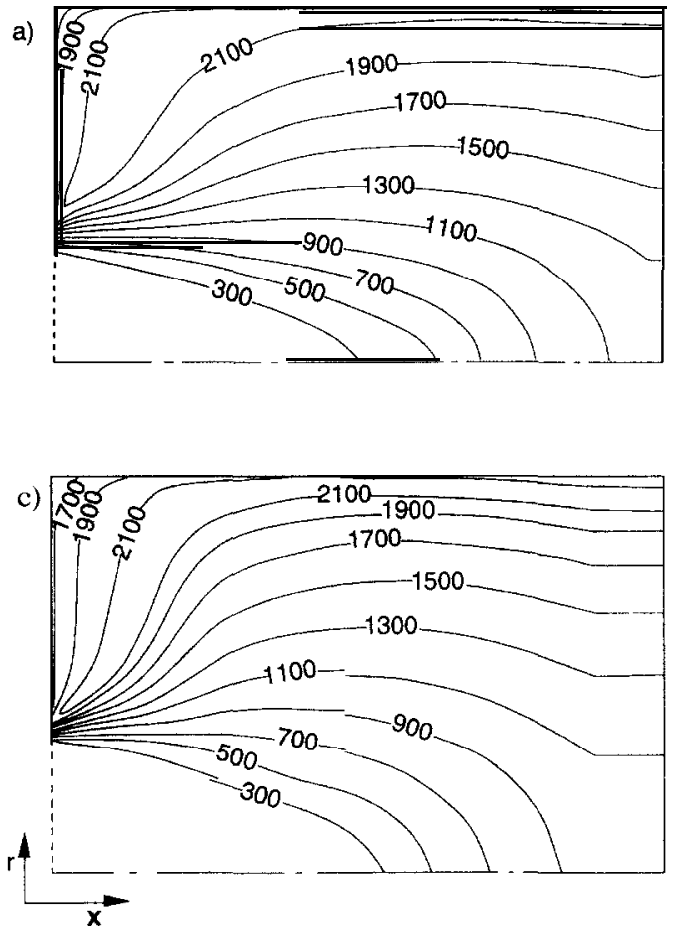

b)

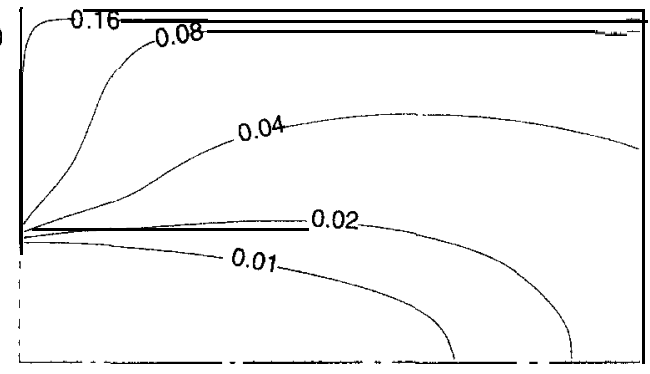

d)

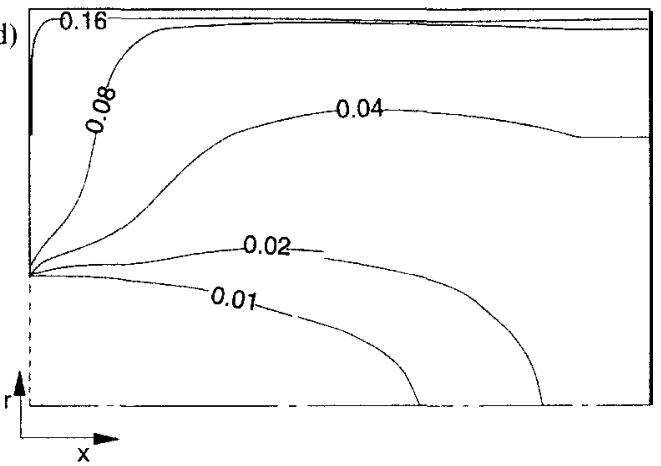

Fig. 7. $m$ Predicted contours for the flow in a solid fuel combustion chamber. a) Temperature (K). Single-block grid. b) Mixture fraction, Single-block grid. c) Temperature (K), Multi-block grid. d) Mixture fraction. Multi-block grid. 

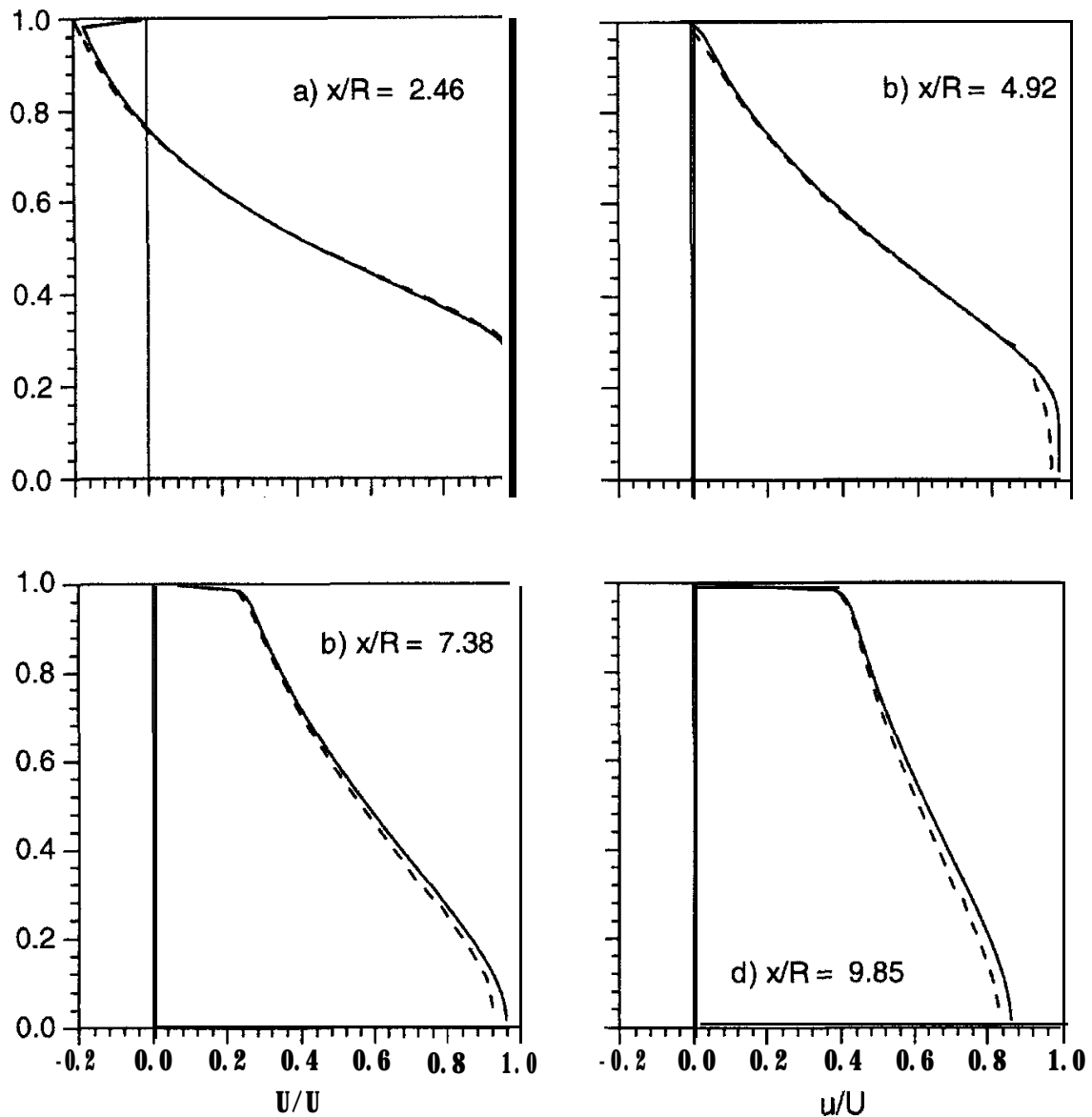

Fig. 8. - Predicted w-velocity profiles for the flow in a solid fuel combustion chamber (solid line: single-block grid, dashed line: multi-block grid).

Calculations were performed using a single-block grid with $80 \times 60$ grid nodes (see Fig. 6a) and a three-block grid with discontinuous grid lines along the interblock boundaries, which is shown in Figure 6 b. As in the previous case, additional computations were carried out using a multi-block grid with continuous grid lines along the interblock boundaries and with the grid nodes coincident with those of the single blockgrid. Since no noticeable differences were observed as compared to the single-block predictions, the results obtained using this multi-block grid are not reported here. In this problem, the number of iterations required to achieve convergence in the single-block and multiblock calculations with continuous grid lines along the interblock boundaries is about the same.

Again, small underelaxation factors were used for $k$ and $\varepsilon$ equations during some iterations at the beginning of the iterative procedure. The reasons given for the isothermal turbulent flow case still hold. Because of the change of density in the reactive flow case, the problem is more critical, and 150 iterations were performed before increasing the $k$ and $\varepsilon$ underrelaxation factors. The iteration number chosen for changing the $k$ and $\varepsilon$ underrelation factors (30 in the previous problem, 150 in the present one) is given as an input. The code automatically uses underrelaxation factors for $k$ and $\varepsilon$ equal to $1 / 10$ of the input values at the beginning and switches to the input values at that iteration.

Figure 7 shows the predicted temperature and mixture fraction contours for the two grids mentioned above. The mixture fraction contours are very similar for the two grids and exhibit the expected trend. Very low values of mixture fraction, characteristic of a lean mixture, are observed in the core of the combustor, and large values, typical of a rich mixture, are confined to a thin annular region close to the wall. The reaction zone corresponds to the region where higher temperatures occur. In the single-block calculations a larger region of temperatures above $2100 \mathrm{~K}$ was obtained which extends closer to the step at the inlet.

Predicted radial profiles of the normalized ILvelocity component at four stations are plotted in Figure 8. Since no velocity data is available for this problem, only the predicted results are shown. The profiles obtained for the two grids are similar to each other, with larger differences occuring at the centreline, for stations far from the inlet section. The single block grid yields slightly larger velocities 
which are consistent with the higher temperatures and, therefore, lower densities obtained in that case.

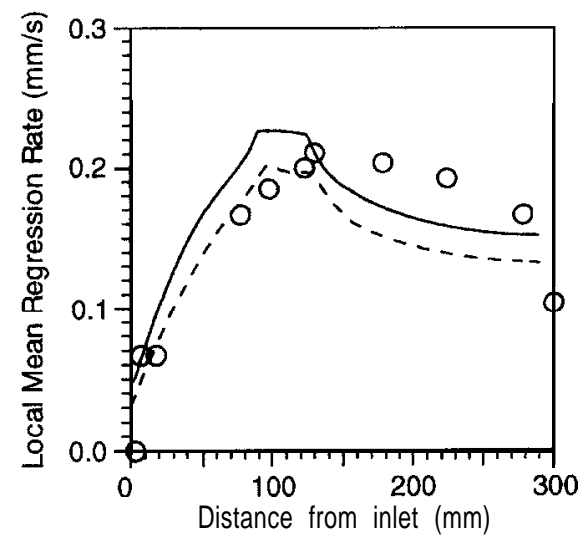

Fig. 9. - Predicted and measured regression rate along the wall (symbols: measurements [ 13], solid line: single-block grid, dashed line: multi-block grid).

The predicted evolution of the local mean regression rate along the fuel wall is plotted in Figure 9 along with the experimental data [ 13]. The predicted regression rate increases significantly along the centreline up to the reattachment point, and decreases farther downstream approaching a constant value. In the region of the flow upstream of the reattachment point there is a slight overprediction of the regression rate. Farther downstream the predicted local mean regression rate decreases and tends to underestimate the experiments which show only a very smooth peak. The overprediction of the local mean regression rate in the recirculation region is attributed to the inability of the $k-\varepsilon$ model, together with the wall functions, to predict accurately the heat transfer behind a step [13]. Since the regression rate is linearly related to the heat flux to the wall (see equation 23), the regression rate is affected by the errors associated with the heat flux calculation.

The use of wall functions requires that the grid nodes where the wall boundary conditions are applied, i.e., the grid nodes associated with control volumes with a cell face coincident to the wall, are placed in the log-layer. The location of the log-layer is not known a priori but the program automatically generates a warning if a boundary point does not lie in the loglayer. This often happens at the beginning of the iterative procedure, but the warnings generally tend to disappear as convergence is approached. This mean that, in general, for the grids that have been used, the boundary points are placed in the region of validity of the laws of the wall. Therefore, errors associated with the laws of the wall result from the assumptions inherent to those laws rather than to their application to flow regions where they do not hold.

The two runs yielded qualitatively similar evolutions of the local mean regression rate along the wall, but the single-block predictions are consistently higher than the multi-block ones. This is in agreement with the higher temperatures obtained in the single-block case. In fact, if the temperature is higher, the heat flux increases and so does the blowing velocity of the fuel. However, both evolutions exhibit the shortcomings described above if compared to the experimental data.

\section{V - CONCLUSION}

A computer code for the calculation of turbulent reactive flows in a solid fuel combustion chamber of a ramjet was successfully extented to accommodate a multi-block domain decomposition technique. This technique allows fine grid resolution to be restricted to regions of large gradients, while a coarser grid may be used elsewhere. Multi-block grids both with and without grid line continuity across the interblock boundaries were used, to allow the comparison between single-block and multi-block results.

In the case of isothermal or reactive turbulent flow multi-block calculations it is necessary to start the iterations with small values of the underrelaxation factors for the turbulent kinetic energy and dissipation rate equations, in order to prevent divergence of the iterative solution procedure. These factors may be increased after a relatively stable solution has been achieved.

The results obtained show that, for the test cases studied here, the numerical solutions are identical using a single-block grid and a three-block grid with continuous grid lines along the interblock boundaries and with grid nodes coincident with those of the single-block grid. If there are grid line discontinuities along the interblock boundaries, flux conservation is maintained, due to the conservative interblock boundaries treatment.

Calculations peformed for a turbulent flow through a sudden pipe expansion were in good agreement with the data in the reattachment region, but a slower redevelopment of the flow was predicted farther downstream. The code was also applied to a solid fuel combustion chamber of a ramjet. In this case, the evolution of the local regression rate along the wall was in qualitative agreement with the data, but quantitative disagreement was found. The observed discrepancies are mainly due to the shortcomings of the turbulence model employed.

\section{REFERENCES}

[1] Schulte G. - Fuel Regression and Flame Stabilization Studies, J. Propulsian and Pow er, 2, N o. 4, 1986, 301-304.

[2] Schulte G., Pein R., Högl A. - Temperature and Concentration Measurements in a Solid Fuel Ramjet Combustion Chamber, J. Propulsion and Power, 3, No.

2, 1987, 114-120. 
[3] Zvuloni R., Gany A., Levy Y. - Geometric Effects on the Combustion in Solid Fuel Ramjets, J. Propulsion and Power, 5, No. 1, 1986, 32-37.

[4] Zvuloni R., Levy Y., Gany A. Investigation of a Small Solid Fuel Ramjet Combustor, J. Propulsion and Power, 5, No. 3, 1989, 269-275.

[5] Ben-Arosh R., Gany A. - Similarity and Scale Effects in Solid-Fuel Ramjet Combustors, J. Propulsion and Power, 8, No. 3, 1992, 615-623.

[6] Stevenson C. A., Netzer D. W. - Primitive Variable Model Applications to Solid-Fuel Ramjet Combustion, J. Spacecraft and Rockets, 18, No. 1, 1981, 89-94.

[7] Milshtein T., Netzer D. W. - Three-Dimensional, Primitive-Variable Model for Solid-Fuel Ramjet Combustion, J. Spacecraft and Rockets, 23, N o. 1, 1986, 113-1 17.

[8] Vos J. B. - The Calculation of Turbulent Reacting Flows with a Combustion Model Based on Finite Chemical Kinetics, Ph.D. thesis, Delft University of Technology, Delft, The Netherlands, 1987.

[9] Vos J. B. Calculating Turbulent Reacting Rlows Using Finite Chemical Kinetics, AIAA J., 25, 1987, 13651372

[10] van der Geld C. W. M., Korting P. A. 0. G., Wijchers T. Combustion of PMMA, PE and PS in a Ramjet, Combustion and Flame, 79, 1990, 299-306.

[ 11] Korting P. A. 0. G., van der Geld C. W. M., Wijchers T., Schöyer H. F. R. - Combustion of Polymethylmethacrylate in a Solid Fuel Ramjet, J. Propulsion and Power, 6, 1990, 263-272.

[ 12] Elands P. J. M., Korting P. A. 0. G., Veraar R. G., Dijkstra F. Theoretical and Experimental Performance of a Solid Fuel Ramjet Combustor Cycle for Hypersonic Flight Conditions, AGARD CP-479, Paper No. 29, 1990.

[13] Elands P., Korting P., Wijchers T., Dijkstra F. Comparison of Combustion Experiments and Theory in Polyethylene Solid Fuel Ramjets, J. Propulsion and Power, 6, 1990, 732-739.

[14] Rai M. M. - A Conservative Treatment of Zonal Boundaries for Euler Equation Calculations, AIAA paper 84-0164, 1984.

[15] Chieng C. C., Launder B. E. On the Calculation of Turbulent Heat Transport Downstream from an Abrupt Pipe Expansion, Numerical Heat Transfer, 3, 1980, 189-207.
[16] Patankar S. V., Spalding D. B. - Heat and Mass Transfer in Boundary Layers, 2nd ed., Intertext Books, London, 1970.

[ 17] Patankar S. V. - N umerical Heat Transfer and Fluid Flow, Hemisphere Publishing Corporation, New York, 1980.

[18] Shyy W., Liu J., Wright J. - Pressure-Based Viscous Flow Computation Using Multiblock Overlapped Curvilinear Grids, Numerical Heat Transfer, Part B, 25, 1994, 39-59.

[19] Chaturvedi M. C. - Flow Characteristics of Axisymmetric Expansions, J. of the Hydraulics Division, Proc. ASCE, 89, 1963, 61-92.

[20] Vos J. B., van Dijk J. H. The Development of a Computational Model for a 2 Dimensional Turbulent Flow. Part II: Description of the Computer Code and Computational Results of Various Pipe Flows, Report LR-469, PML 1985-C22, Delft/Rijswijk, The Netherlands, 1985.

[21] Nallasamy M. - Turbulence Models and Their Applications to the Predictions of Internal Flows: a Review, Computers and Fluids, 15, No. 2, 1987, 151-194.

[22] Back L. H., Roschke E. J., Shear Layer Flow Regimes and Wall Instabilities and Reattachment Lengths Downstream of an Abrupt Circular Channel Expansion, J. Appl. Mech., 94, 1972, 677-681.

[23] Moon L. F., Rudinger G. - Velocity Distribution in an Abruptly Expanding Circular Duct, J. Fluids Engineering, 99, 1977, 226-230.

[24] Gosman A. D., Khalil E. E., Whitelaw J. H. - The Calculation of Two-Dimensional Turbulent Recirculating Flows, Proc. Turbulent Shear Flow s, 1, 1980, 237-255.

[25] Ha Minh H. Chassaing P. - Perturbations of Turbulent Pipe Flow, Proc. Turbulent Shear Flows, 1, 1980, 178-197.

Acknowledgments. - This work has been supported by TNO, Prins Maurits Laboratory, in The Netherlands, within the framework of the workprogramme "Improvements and Updates of the COPPEF Code", and by the Portuguese Ministry of Defense. 